Published in final edited form as:

JAMA Cardiol. 2017 March 01; 2(3): 314-318. doi:10.1001/jamacardio.2016.4652.

\title{
Diabetes, kidney disease and cardiovascular outcomes in the Jackson Heart Study
}

\author{
Maryam Afkarian, MD-PhD, \\ Kidney Research Institute and Division of Nephrology, University of Washington, Seattle, WA \\ Ronit Katz, DPhil, \\ Kidney Research Institute and Division of Nephrology, University of Washington, Seattle, WA \\ Nisha Bansal, MD, \\ Kidney Research Institute and Division of Nephrology, University of Washington, Seattle, WA
} Adolpho Correa, MD, MPH, PhD, MBA, Jackson Heart Study, Department of Medicine, University of Mississippi Medical Center, Jackson, MS

\section{Bryan Kestenbaum, MD, MS,} Kidney Research Institute and Division of Nephrology, Department of Medicine, University of Washington, Seattle, WA

\section{Jonathan Himmelfarb, MD,}

Kidney Research Institute and Division of Nephrology, University of Washington, Seattle, WA

lan H. de Boer, MD, MS, and

Kidney Research Institute, Division of Nephrology, Veterans Affairs Puget Sound, University of Washington, Seattle, WA

\section{Bessie Young, MD, MS}

Kidney Research Institute, Division of Nephrology, Veterans Affairs Puget Sound, University of Washington, Seattle, WA

\section{Abstract}

Background and objectives-African Americans have high rates of cardiovascular disease and mortality. Diabetes and chronic kidney disease (CKD), risk factors for cardiovascular mortality in the general population, are common among African Americans. Our objective was to assess the contribution of diabetes and CKD to cardiovascular disease (CVD) and mortality in African Americans.

Design, setting, participants and measurements-This is an observational cohort study of 3,211 participants in the Jackson Heart Study (JHS), where rates of incident stroke, incident coronary heart disease (CHD) and cardiovascular mortality were quantified in people with

Corresponding author: Maryam Afkarian, MD-PhD, Box 359606, $3259^{\text {th }}$ Ave, Seattle, WA 98104. Telephone: (206) 685-3168 Fax: (206) 685-9399. afkarian@u.washington.edu. 
diabetes, CKD (defined as estimated GFR $<60 \mathrm{ml} / \mathrm{min} / 1.73 \mathrm{~m}^{2}$, urine albumin-to-creatinine ratio (ACR) $230 \mathrm{mg} / \mathrm{g}$, or both) or both.

Results-Among the participants, 456 (14.2\%) had only diabetes, 257 (8.0\%) only CKD, 201 (6.3\%) both and 2,297 (71.5\%) neither. Diabetes without CKD was associated with excess risks of incident stroke, incident CHD and cardiovascular mortality after adjustment for age, gender, income, smoking, hypertension, hyperlipidemia and history of CVD (excess incidence rates of 2.6, 2.6 and 2.4 per 1000 person-years, p 0.015, 0.027, 0.016, respectively). CKD without diabetes was associated with comparable non-significant excess risks for incident stroke and CHD (2.5 and 2.4 per 1000 person-years, $p>0.05$ ), but a larger increase in cardiovascular mortality (7.3 per 1000 person-years, $\mathrm{p}=0.001)$. The combination of diabetes and $\mathrm{CKD}$ was associated with greater excess risks of incident stroke (13.8 per 1000 person-years, $\mathrm{p}=0.001)$, CHD (12.8 per 1000 person-years, $\mathrm{p}=0.002)$ and cardiovascular mortality ( 14.8 per 1000 person-years, $\mathrm{p}<0.001)$. The excess risks associated with the combination of diabetes and CKD were larger than those associated with other established risk factors, including prevalent CVD.

Conclusions-The combination of diabetes and kidney disease is associated with substantial excess risks of cardiovascular events and mortality among African Americans.

\section{Keywords}

diabetes; CKD; stroke; coronary heart disease; cardiovascular mortality; African American

\section{INTRODUCTION}

African Americans experience a higher rate of cardiovascular disease than Caucasians.(1) African Americans residing in certain regions of the US, such as Mississippi, have even higher rates of cardiovascular mortality than those residing elsewhere in the country.(2-4) Diabetes and chronic kidney disease (CKD) are both significant risk factors for cardiovascular disease and mortality. In fact, cardiovascular disease is the predominant cause of mortality among people with diabetes and kidney disease.(5, 6) Previous studies, mostly in Caucasian or ethnically mixed populations, have shown that CKD is a powerful predictor of the excess mortality in diabetes.(7-9) Using data from National Health and Nutrition Examination Survey (NHANES III), we previously reported that among people with diabetes, those with kidney disease have the highest cumulative incidence of 10-year mortality. Interestingly, absent kidney disease, mortality in people with diabetes was not drastically higher than that of a reference population without diabetes or kidney disease.(9)

African Americans are 1.7 times more likely than Caucasians to be diagnosed with diabetes, (10) 2.4 times more likely to develop end-stage kidney disease due to diabetes(11) and 2.2 times more likely to die as a result.(12) In this study, we asked whether kidney disease is predictive of cardiovascular outcomes among African Americans. To address this, we examined the association between diabetes and kidney disease and excess risk for incident stroke and coronary heart disease (CHD), as well as cardiovascular mortality, in the Jackson Heart Study (JHS) population. 


\section{MATERIALS AND METHODS}

\section{Study population}

The Jackson Heart Study (JHS) is a single-site, community-based, prospective cohort study of risk factors and course of cardiovascular disease in non-institutionalized adult African Americans. Between 2000-2004, the study enrolled 5,301 African Americans aged 21-94 years, residing in three counties within Mississippi Metropolitan Statistical Area (Hinds, Madison and Rankin). During this interval, the baseline data were collected using a selfadministered questionnaire, an in-home interview and a clinic visit. The current study used data from 3,211 JHS participants, excluding participants with missing data on baseline diabetes status $(\mathrm{N}=61)$, serum creatinine $(\mathrm{N}=34)$, serum cystatin $\mathrm{C}(\mathrm{N}=81)$, urine albumin and creatinine $(\mathrm{N}=1,914)$. Follow-up clinical examinations were conducted in 4-year intervals, with exam 2 conducted between 2005 to 2008 and exam 3 between 2009 to 2012 . Median follow-up duration was 6.99 years. In addition, interim medical events and vital statistics were also collected using annual telephone interviews. The study was approved by the institutional review boards of Jackson State University, Tougaloo College and the University of Mississippi Medical Center. All of the participants gave written informed consent. The use of de-identified data for this study was considered non-human subjects research by the Human Subjects Division of the University of Washington.

\section{Diabetes definition}

Diabetes was defined as self-reported diabetes, hemoglobin A1c $\succeq 6.5 \%,(13)$ fasting glucose $>126 \mathrm{mg} / \mathrm{dl}$, as per 2010 American Diabetes Association guidelines,(14) or use of glucoselowering medications at baseline exam. Glycated hemoglobin (HbA1c) concentration was measured using a high-performance liquid chromatography system (Tosoh Corporation, Tokyo, Japan) in blood samples collected after an overnight fast.

\section{Chronic kidney disease definition}

CKD was defined as albuminuria, reduced estimated glomerular filtration rate (eGFR), or both, using data from the baseline visit. Albuminuria was defined as a urine albumin to creatinine ratio (ACR) $\geq 30 \mathrm{mg} / \mathrm{g}$. Reduced eGFR was defined as an eGFR $\$ 0 \mathrm{ml} / \mathrm{min} /$ $1.73 \mathrm{~m}^{2}$. Urine albumin and creatinine concentrations were measured in clean-catch random $(\mathrm{N}=2209)$ or 24-hour $(\mathrm{N}=1002)$ urine samples, collected at baseline visit after an overnight fast. There were 276 JHS participants with both 24-hour and spot urine ACR values with a correlation of 0.965. Urine albumin was measured using a human albumin kit (Dade Behring, Neward, Delaware) on a Dade Behring BN II nephelometer. Urine creatinine was measured at the University of Mississippi Medical Center Laboratory Reading Center using a multi-point enzymatic spectrophotometric assay (Vitros CREA dry reaction slides on a Vitros 950 Ortho-Clinical Diagnostics Analyzer, Raritan, New Jersey). Creatinine concentrations were calibrated to the Cleveland Clinic-equivalent Minnesota Beckman CX3 assay. Serum creatinine was measured using the Jaffe method and calibrated to measurements traceable to isotope dilution mass spec (IDMS). Serum cystatin C was measured by a particle-enhanced immunonephelometric assay (N Latex Cystatin C, Siemens AG, Munich). Estimated GFR was calculated from serum concentrations of creatinine and cystatin $\mathrm{C}$ measured at baseline using the 2012 CKD-EPI equation. 


\section{Other characteristics}

Demographic and socioeconomic variables (age, gender, income and education) as well as smoking history (never, former, current) were obtained during the baseline interview. Income was derived from family income and size, adjusted by the year of data collection to account for inflation and categorized in four groups (Table 1). Medications used in the two weeks preceding the interview were brought to the clinic and transcribed from bottles and coded by pharmacists using the Medispan dictionary. Blood pressure was measured by trained staff in seated participants after a 5-minute rest, using an appropriately sized cuff and a Hawksley random-zero sphygmomanometer (Hawksley and Sons, Ltd). Two blood pressure readings were taken one minute apart and the arithmetic average was recorded. Hypertension was defined as systolic blood pressure $\geq 140 \mathrm{mmHg}$, diastolic blood pressure $\geq 90 \mathrm{mmHg}$ and/or use of antihypertensive medications. Total cholesterol and triglyceride concentrations were measured in fasting blood samples using the Roche enzymatic methods using a Cobras centrifuge analyzer (Hoffman-La Roche). Low-density lipoprotein (LDL) cholesterol concentrations were estimated using the Friedewald formula.(15) Hyperlipidemia was defined as a low-density lipoprotein cholesterol (LDL) $\geq 160 \mathrm{mg} / \mathrm{dL}$ and/or use of lipid-lowering medications

\section{Outcomes}

The outcomes were incident coronary heart disease, incident stroke and mortality from cardiovascular causes. These outcomes were captured during the annual telephone interviews with participants and their family members, as well as JHS exam 2 and exam 3. During the interviews and exams, trained staff identified interim medical events, including new health events, diagnostic tests, hospitalizations, new diagnoses or death. These were subsequently confirmed by review of medical records, including discharge summaries, ICD-9 codes and procedure codes. Cohort deaths were additionally identified from the monthly printout of Mississippi state department of health, systematic review of death certificates, hospital chart review, use of obituary notices and linkage to the National Death Index. Final classification of all cardiovascular events required medical chart review and adjudication by trained physicians.

Incident coronary heart disease was defined as myocardial infarction or need for coronary revascularization, based on data abstracted from medical records, which included presenting symptoms, relevant clinical data (cardiac biomarkers, electrocardiogram, etc) and diagnostic and therapeutic procedures. Adjudicating physicians assigned a diagnosis of no, probable, or definite myocardial infarction based on the abstracted data. For these analyses, probable or definite myocardial infarction was used as part of the coronary heart disease outcome. Incident stroke at outpatient or inpatient settings was defined as cerebrovascular accident due hemorrhagic or ischemic stroke based on review of medical records, including pertinent diagnostic and therapeutic procedures by qualified adjudicating physicians. An incident stroke defined as probable or definite based on this analysis was classified as an event for this outcome. Causes of death were identified by review of ICD-9 codes for the underlying and contributory causes of death, physician (and when indicated coroner or medical examiner) questionnaires, interviews with the next-of-kin and/or any non-family witnesses of death. Deaths from cardiovascular causes were ascertained by review of the causes of 
death by three physicians (authors in this manuscript: M.A., N.B. and B.K.) and consisted of XX.

\section{Statistical methods}

We categorized participants by presence or absence of diabetes and CKD, and compared the distribution of demographics and covariates in these four mutually exclusive groups. Participants were considered at risk for each of the outcomes from their baseline visit until the first occurrence of the outcomes or censoring due to loss to follow-up, the end of available follow-up, or death due to non-cardiovascular causes. Participants with a prior stroke were excluded from the analyses where outcome was incident stroke. Participants with a prior CHD were excluded from the analyses where the outcome was incident CHD. However, participants with prior stroke or CHD were included in the analyses where the outcome was CV mortality. Incidence rates (per 1000 person-years with $95 \%$ confidence intervals) for incident stroke, incident coronary heart disease or cardiovascular mortality were calculated using Poisson regression in four groups: participants with no diabetes or CKD, diabetes but no CKD, CKD but no diabetes and both diabetes and CKD. Risk differences were estimated by comparing incidence rates in each group to that in the reference group (participants with no diabetes or CKD) from the Poisson regression and the 95\% confidence intervals for the risk differences were computed from a bootstrap analysis of 1000 samples. Cox regression was used to estimate the relative hazard of each outcome, adjusting for relevant covariates, in the same four groups. In each analysis, models were first adjusted for age, age squared, gender and income (model 1). Given the large age range, adjustment for age was done using both simple and quadratic terms. Income was binarized by collapsing the four categories into two categories of low (combination of poor and lowermiddle) and high (combination of upper-middle and affluent). Education was not included in the final model because it did not remain significant in presence of income. The final model was additionally adjustment for smoking status, hypertension, hyperlipidemia and prevalent cardiovascular disease (model 2). TEXT DESCRIBING CALCULATION OF INTERACTIONS

All analyses were conducted in STATA version 13 (College Park, Tx, StataCorp LP) and SPSS version 22 (Armonk, NY: IBM Corp) and p-values < 0.05 were considered statistically significant.

\section{RESULTS}

\section{Characteristics of study subjects}

Of the 5,301 JHS participants, we excluded those with missing measures of diabetes ( $\mathrm{n}=61)$, serum creatinine $(n=34)$ or cystatin $(n=81)$ and urine albumin-to-creatinine ratio (ACR, $\mathrm{n}=1,914$ ), leaving 3,211 who were included in this study (Supplementary Figure 1). Among the study population, $456(14.2 \%)$ had only diabetes, 257 (8.0\%) only CKD, $201(6.3 \%)$ both and 2,297 (71.5\%) neither at study entry (Table 1). Majority of the participants with CKD had albuminuria (Supplementary Figure 2). Participants with diabetes and/or CKD were older and more obese. Presence of CKD and diabetes was associated with higher prevalence of hypertension and use of antihypertensive medications. Despite higher 
prevalence of antihypertensive use, the mean systolic blood pressures were higher in subgroups with CKD. More participants with diabetes were using HMG-CoA reductase inhibitors. However, total cholesterol as well as low-density lipoprotein cholesterol concentrations were comparable with or without CKD or diabetes. Participants with diabetes and/or CKD included more people with lower incomes. In addition, diabetes and CKD were more common in people with lower income: CKD was present in $16 \%$ of people in the combined poor and lower-middle categories vs. $9 \%$ in the combined upper-middle and affluent categories. Diabetes was present in $25 \%$ of people in the combined poor and lowermiddle categories vs. $14 \%$ in the combined upper-middle and affluent categories.

\section{Cardiovascular outcomes by diabetes status}

The incidence rate of stroke in was higher in JHS participants with diabetes than those without (1.3 vs. 6.5 per 1000 person-years), after adjustment for socio-demographic and clinical covariates (Table 2). Similarly, incident coronary heart disease (CHD) and cardiovascular mortality were more frequent in those with diabetes than without (1.9 vs. 6.9 and 1.3 vs. 6.0 per 1000 person-years).

\section{Cardiovascular outcomes by CKD and diabetes status}

In absence of diabetes and CKD, the unadjusted rate of incident stroke was 1.7 per 1000 person-years (95\% confidence interval, CI, 1.1 to 2.4) (Table 3). In presence of diabetes alone, CKD alone or diabetes and CKD together, this rate increased to 5.7 (95\% CI 2.1 to 9.8), 5.9 (95\% CI 2.1 to 9.8), and 17.0 (95\% CI 9.4 to 24.7) per 1000 person-years, respectively. Compared with the reference group without either diabetes or CKD, presence of diabetes only was associated with an excess risk for incident stroke of 2.6 (95\% CI, 0.5 to 4.7) per 1000 person-years, after adjustment for socio-demographic and clinical variables. CKD alone was associated with an excess risk of 2.5 (95\% CI -0.1 to 5.2) per 1000 personyears for stroke, which lost significance after adjustment for socio-demographic variables. The combination of diabetes and CKD was associated with an excess risk for incident stroke of 13.8 (95\% CI 5.3 to 22.3 ) per 1000 person-years in fully adjusted analyses.

Compared with the reference group, diabetes alone was associated with an excess risk for incident CHD of 2.6 (95\% CI 0.3 to 4.8) per 1000 person-years, after full adjustment. The combination of diabetes and CKD was associated with an excess risk for incident CHD of 12.6 (95\% CI 4.9 to 20.8) per 1000 person-years, after full adjustment. Presence of diabetes alone, CKD alone or the combination of diabetes and CKD was associated with excess risks for cardiovascular mortality of 2.4 (95\% CI 0.4 to 4.3 ), 7.3 (95\% CI 3.0 to 11.5) and 14.8 ( $95 \%$ CI 7.2 to 22.3) percent per year after full adjustment. Modeling time to the first event using the Cox proportional hazard model showed similar results, including 3.3-fold increase in incident CHD (95\% CI 1.79-6.20), 6.23-fold (95\% CI 3.22-12.09) increase in incident stroke and 6.4-fold (95\% CI 3.53-11.78) increase in CV mortality (Table 4). There was a trend towards additive interaction between the risk differences associated with diabetes and CKD for incident stroke, incident CHD and CV mortality (synergy index 0.87, 0.78 and 0.52 , respectively). However, only the interaction for incident stroke attained statistical significance (p-value 0.004 ), likely due to limited power. 


\section{The excess risk of cardiovascular outcomes associated with CKD and diabetes, compared with other clinical risk factors}

To compare the increase in risk of the $\mathrm{CV}$ outcomes associated with diabetes and CKD to that associated with other cardiovascular risk factors (particularly prevalent CVD), we examined the risk differences for each risk factor in the fully adjusted model (Model 2, Table 3). However, prevalent CVD is defined differently for each outcome. For incident stroke, prevalent CVD consisted of prevalent CHD because the participants with prevalent stroke had to be excluded. Similarly, for the outcome of incident CHD, prevalent CVD consisted of prevalent stroke. Only for the outcome of CV mortality did prevalent CVD include both prevalent stroke and CHD. In the fully adjusted model, the combination of diabetes and CKD was associated with a greater increase in the risk of incident stroke, incident CHD and cardiovascular death than other cardiovascular risk factors, including prevalent cardiovascular disease, hyperlipidemia, hypertension and smoking. Diabetes and CKD individually were associated with comparable increases in rate of incident stroke and CHD, increases which were of similar magnitude to those for hypertension and smoking, but less than that of prevalent cardiovascular disease. However, CKD alone was associated with a much greater increase in rate of cardiovascular mortality than diabetes alone, an increase which was comparable to that of prevalent cardiovascular disease (Figure 1).

\section{DISCUSSION}

In an African American population with high incidence of cardiovascular disease and mortality, diabetes alone was associated with excess risks of incident stroke, CHD and cardiovascular mortality, while CKD alone was associated with excess risk for cardiovascular mortality. However, the combination of diabetes and kidney disease was associated with a greater excess risk for incident stroke, CHD and cardiovascular mortality than established clinical risk factors, including a history of cardiovascular disease.

African Americans, particularly in the Southern low-income rural communities, have some of the highest rates of all-cause and cardiovascular mortality in the US. Kidney disease and diabetes, both risk factors for cardiovascular disease, are disproportionately more common in this population. CKD is associated with increased risk of all-cause and cardiovascular mortality both in the general population (16-20) and among people with diabetes. In a nationally representative population, kidney disease captured a majority of the excess mortality risk associated with diabetes.(9) Furthermore, absent kidney disease, diabetes was not associated with a large increase in mortality. Here, we extend that work by examining the association of diabetes and kidney disease with cardiovascular mortality, but also incident cardiovascular events, in a high-risk African American population. Risk differences were evaluated on an absolute scale in order to present the clinically relevant marginal risk associated with diabetes, kidney disease or the combination, over the baseline risk among the reference population without diabetes or CKD. Using this approach, we found a much larger excess risk for cardiovascular mortality in presence of CKD than diabetes, consistent with our findings in NHANES. Similarly, the combination of diabetes and CKD was associated with a much larger excess risk for cardiovascular mortality than either risk factor 
alone. Consistently, we found a trend toward an additive interaction between CKD and diabetes, but this did not reach statistical significance.

On the other hand, while CKD alone conferred a significant excess risk for cardiovascular mortality, it did not significantly increase the risk for incident stroke or CHD. The combination of diabetes and kidney disease was associated with a significant excess risk for both incident stroke and CHD. However, each risk factor alone was associated with a small excess risk for these outcomes, which in the case of kidney disease was not robust to adjustment, suggesting an additive interaction between diabetes and CKD. This observation raises the possibility that in absence of diabetes, CKD may contribute more to reduced survival after a cardiovascular event, rather than predisposition to it. In fact, presence and severity of kidney disease is associated with lower survival after cardiovascular events, including acute myocardial infarction, stroke or congestive heart failure.(21) In an interesting parallel, even though African Americans have comparable rates of cardiovascular events to Caucasians, $(22,23)$ they experience higher cardiovascular mortality than Caucasians.(24-26) This suggests that the higher prevalence of kidney disease in African Americans may contribute to the disparity in cardiovascular mortality in this population, relative to Caucasians.

The gap in socioeconomic status is believed to at least partly underlie the disparity in cardiovascular outcomes and mortality between African Americans and Caucasians.(REF) In JHS, both diabetes and kidney disease were more prevalent in participants with lower socioeconomic status, as assessed by income. Adjustment for age, gender and socioeconomic status attenuated the excess risk for cardiovascular outcomes in presence of the combination of diabetes and kidney disease. However, most of this attenuation was due to age (and not socio-economic status). Furthermore, even after this adjustment, the excess risk for cardiovascular outcomes in presence of concomitant diabetes and kidney disease remained sizable and significant. On the other hand, after adjustment for both diabetes and kidney disease, the excess risk associated with income was not significant for incident stroke and CHD, but remained significant (though small) for cardiovascular mortality. These observations suggest that the previously observed associations between socioeconomic status and CVD outcomes may be mediated via higher rates of diabetes and CKD in people with lower socioeconomic status. They further suggest that socioeconomic status, similar to $\mathrm{CKD}$, may have a greater influence on survival after a cardiovascular event, than the predisposition to it.

Our findings are also relevant to the question of risk equivalence for the risk of CV outcomes associated with CKD, compared to that from diabetes or prevalent CVD. Previous work in largely Caucasian populations has shown that CKD contributes significantly to cardiovascular risk stratification(27) (27) and that combination of diabetes and CKD is associated with comparable rates of incident myocardial infarction to pre-existing coronary heart disease.(28)(28) Given our focus on incident outcomes (stroke and CHD), prevalent stroke (or CHD) could not be included in our assessment of risk factor for incident stroke (or CHD). As such, our findings address this question more fully for CV mortality, where prevalent CVD included prevalent stroke and CHD. Consistent with prior studies,(27) we found that in this African American cohort, kidney disease, particularly in presence of 
diabetes, was associated with a greater excess risk of cardiovascular outcomes and mortality than other clinically utilized cardiovascular risk factor, including pre-existing cardiovascular disease. This finding has direct clinical implications for cardiovascular risk stratification and intensive therapeutic targeting of the subpopulations at highest risk for future cardiovascular events and mortality. For example, as pointed out in previous studies, full assessment of kidney function, including not only estimated GFR but also urine albumin excretion, is likely to be beneficial for complete cardiovascular risk assessment.

Interestingly, the incidence of stroke was comparable to that of coronary heart disease in JHS. This is counter to the general population, where incidence of coronary heart disease equals or exceeds that of all other cardiovascular events together. This may be related to the higher incidence of stroke noted in the Southeastern US, compared to the rest of the country. The higher rates of stroke observed in African Americans, vs. Caucasians, may also contribute to this finding.

The observational nature of the study precludes differentiation of whether the combination of diabetes and kidney disease are causally related to cardiovascular outcomes, even though this possibility is strongly supported by pre-existing data. Additional limitations include assessment of kidney function and diabetes at one time-point, small number of events in some categories and potential misclassification in determination of cause of mortality based on ICD codes. The strengths of this study are use of a clinically relevant, high-risk African American population, use of physician-adjudicated cardiovascular outcomes, comparison to population internal references, uniform assessment of diabetes and kidney disease and evaluation of associations on a clinically relevant additive scale.

In conclusion, this study highlights the significance of kidney disease as a risk factor for cardiovascular disease and mortality in African Americans, particularly in presence of diabetes. Especially among African Americans with diabetes, assessment of kidney function may be a helpful component of cardiovascular risk stratification for identification of a subpopulation for intensive risk modification.

\section{Supplementary Material}

Refer to Web version on PubMed Central for supplementary material.

\section{Acknowledgments}

Dr. Afkarian's effort was supported by grants K23DK089017 and R01DK104706 from the NIDDK and a Norman S. Coplon Extramural Grant from Satellite Healthcare. Dr. de Boer's effort was supported by grants R01DK087726 and R01DK088762 from the NIDDK. Drs. Katz, de Boer, and Young were supported by grant R01DK102134. This study was supported by Dr. Young's National Institutes of Health (NIH) National Institute of Diabetes, Digestive, and Kidney Disease grant 1R01DK102134-01 (Young, Katz, de Boer). The Jackson Heart Study is supported by contracts HHSN268201300046C, HHSN268201300047C, HHSN268201300048C, HHSN268201300049C, HHSN268201300050C from the National Heart, Lung, and Blood Institute and the National Institute on Minority Health and Health Disparities. Drs. Young and de Boer are also supported in part by funding from the Veterans Affairs Puget Sound Health Care System, Seattle WA. Dr. Afkarian is the guarantor of this study and takes responsibility for the findings and interpretations presented in this manuscript. 


\section{References}

1. Ferdinand KC. Coronary artery disease in minority racial and ethnic groups in the United States. The American journal of cardiology. 2006; 97:12A-19A.

2. Taylor HA, Hughes GD, Garrison RJ. Cardiovascular disease among women residing in rural America: epidemiology, explanations, and challenges. American journal of public health. 2002; 92:548-551. [PubMed: 11919049]

3. Taylor HA Jr. Establishing a foundation for cardiovascular disease research in an African-American community--the Jackson Heart Study. Ethnicity \& disease. 2003; 13:411-413. [PubMed: 14632260]

4. Murray CJ, Kulkarni SC, Michaud C, Tomijima N, Bulzacchelli MT, Iandiorio TJ, Ezzati M. Eight Americas: investigating mortality disparities across races, counties, and race-counties in the United States. PLoS medicine. 2006; 3:e260. [PubMed: 16968116]

5. de Boer IH, Katz R, Cao JJ, Fried LF, Kestenbaum B, Mukamal K, Rifkin DE, Sarnak MJ, Shlipak MG, Siscovick DS. Cystatin C, albuminuria, and mortality among older adults with diabetes. Diabetes Care. 2009; 32:1833-1838. [PubMed: 19587367]

6. Ninomiya T, Perkovic V, de Galan BE, Zoungas S, Pillai A, Jardine M, Patel A, Cass A, Neal B, Poulter N, Mogensen CE, Cooper M, Marre M, Williams B, Hamet P, Mancia G, Woodward M, Macmahon S, Chalmers J, Group AC. Albuminuria and kidney function independently predict cardiovascular and renal outcomes in diabetes. Journal of the American Society of Nephrology: JASN. 2009; 20:1813-1821. [PubMed: 19443635]

7. Groop PH, Thomas MC, Moran JL, Waden J, Thorn LM, Makinen VP, Rosengard-Barlund M, Saraheimo M, Hietala K, Heikkila O, Forsblom C. FinnDiane Study G. The presence and severity of chronic kidney disease predicts all-cause mortality in type 1 diabetes. Diabetes. 2009; 58:16511658. [PubMed: 19401416]

8. Orchard TJ, Secrest AM, Miller RG, Costacou T. In the absence of renal disease, 20 year mortality risk in type 1 diabetes is comparable to that of the general population: a report from the Pittsburgh Epidemiology of Diabetes Complications Study. Diabetologia. 53:2312-2319.

9. Afkarian M, Sachs MC, Kestenbaum B, Hirsch IB, Tuttle KR, Himmelfarb J, de Boer IH. Kidney disease and increased mortality risk in type 2 diabetes. J Am Soc Nephrol. 2013; 24:302-308. [PubMed: 23362314]

10. Center for Disease Control and Prevention NCfHS. Summary Health Statistics for US Adults: 2011. 2011.

11. Prevention CfDCa. National Diabetes Surveillance System. 2013.

12. Prevention CfDCa. National Vital Statistics Report. 2013.

13. International Expert Committee report on the role of the A1C assay in the diagnosis of diabetes. Diabetes Care. 2009; 32:1327-1334. [PubMed: 19502545]

14. American Diabetes A. Standards of medical care in diabetes--2010. Diabetes Care. 2010; 33(Suppl 1):S11-61. [PubMed: 20042772]

15. Friedewald WT, Levy RI, Fredrickson DS. Estimation of the concentration of low-density lipoprotein cholesterol in plasma, without use of the preparative ultracentrifuge. Clinical chemistry. 1972; 18:499-502. [PubMed: 4337382]

16. Szczech LA, Best PJ, Crowley E, Brooks MM, Berger PB, Bittner V, Gersh BJ, Jones R, Califf RM, Ting HH, Whitlow PJ, Detre KM, Holmes D. Outcomes of patients with chronic renal insufficiency in the bypass angioplasty revascularization investigation. Circulation. 2002; 105:2253-2258. [PubMed: 12010906]

17. Astor BC, Hallan SI, Miller ER 3rd, Yeung E, Coresh J. Glomerular filtration rate, albuminuria, and risk of cardiovascular and all-cause mortality in the US population. Am J Epidemiol. 2008; 167:1226-1234. [PubMed: 18385206]

18. Bello AK, Hemmelgarn B, Lloyd A, James MT, Manns BJ, Klarenbach S, Tonelli M. Associations among estimated glomerular filtration rate, proteinuria, and adverse cardiovascular outcomes. Clin J Am Soc Nephrol. 6:1418-1426.

19. Hemmelgarn BR, Manns BJ, Lloyd A, James MT, Klarenbach S, Quinn RR, Wiebe N, Tonelli M. Relation between kidney function, proteinuria, and adverse outcomes. Jama. 303:423-429. 
20. Matsushita K, van der Velde M, Astor BC, Woodward M, Levey AS, de Jong PE, Coresh J, Gansevoort RT. Association of estimated glomerular filtration rate and albuminuria with all-cause and cardiovascular mortality in general population cohorts: a collaborative meta-analysis. Lancet. 375:2073-2081.

21. Epidemiology of Kidney Disease in the United States. National Institutes of Health, National Institute of Diabetes and Digestive and Kidney Diseases; Bethesda, MD: 2014. United States Renal Data System: 2014 Annual Data Report.

22. Karter AJ, Ferrara A, Liu JY, Moffet HH, Ackerson LM, Selby JV. Ethnic disparities in diabetic complications in an insured population. JAMA. 2002; 287:2519-2527. [PubMed: 12020332]

23. Lanting LC, Joung IM, Mackenbach JP, Lamberts SW, Bootsma AH. Ethnic differences in mortality, end-stage complications, and quality of care among diabetic patients: a review. Diabetes Care. 2005; 28:2280-2288. [PubMed: 16123507]

24. Dagogo-Jack S. Ethnic disparities in type 2 diabetes: pathophysiology and implications for prevention and management. Journal of the National Medical Association. 2003; 95:774, 779-789. [PubMed: 14527045]

25. Mensah GA, Mokdad AH, Ford ES, Greenlund KJ, Croft JB. State of disparities in cardiovascular health in the United States. Circulation. 2005; 111:1233-1241. [PubMed: 15769763]

26. Gentile NT, Seftchick MW. Poor outcomes in Hispanic and African American patients after acute ischemic stroke: influence of diabetes and hyperglycemia. Ethnicity \& disease. 2008; 18:330-335. [PubMed: 18785448]

27. Matsushita K, Coresh J, Sang Y, Chalmers J, Fox C, Guallar E, Jafar T, Jassal SK, Landman GW, Muntner P, Roderick P, Sairenchi T, Schottker B, Shankar A, Shlipak M, Tonelli M, Townend J, van Zuilen A, Yamagishi K, Yamashita K, Gansevoort R, Sarnak M, Warnock DG, Woodward M, Arnlov J. Consortium CKDP. Estimated glomerular filtration rate and albuminuria for prediction of cardiovascular outcomes: a collaborative meta-analysis of individual participant data. The lancet Diabetes \& endocrinology. 2015; 3:514-525. [PubMed: 26028594]

28. Tonelli M, Muntner P, Lloyd A, Manns BJ, Klarenbach S, Pannu N, James MT, Hemmelgarn BR. Alberta Kidney Disease N. Risk of coronary events in people with chronic kidney disease compared with those with diabetes: a population-level cohort study. Lancet. 2012; 380:807-814. [PubMed: 22717317] 


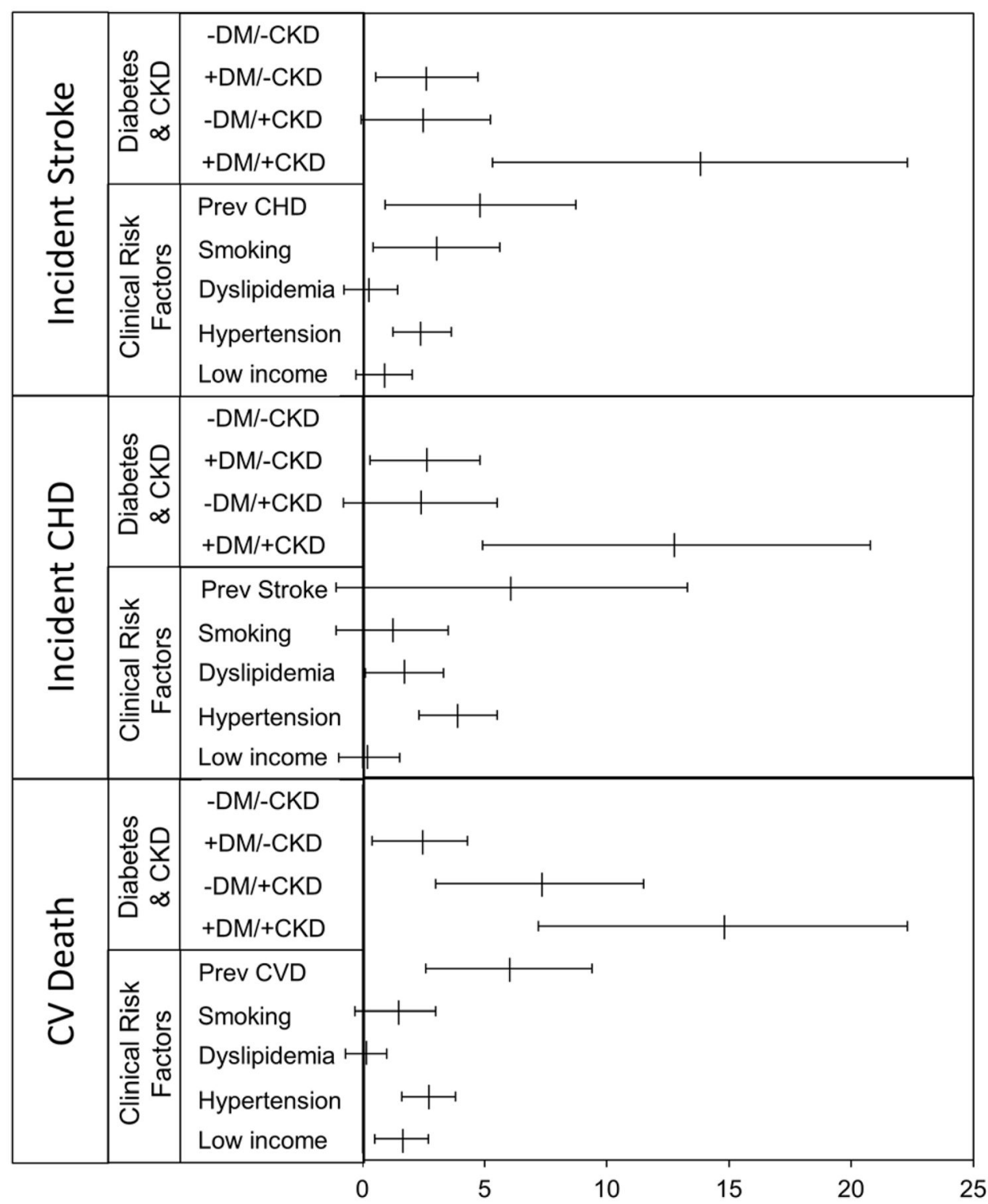

Figure 1. Risk differences for incident stroke, coronary heart disease and cardiovascular mortality (per 1000 person-years) by clinical risk factor in the Jackson Heart Study Incidence rates were calculated using Poisson regression. Absolute risk differences were estimated by comparing the incidence rates in each group to that in the reference group (participants with no diabetes or CKD) using Poisson regression and adjusted for age, age ${ }^{2}$, gender, hypertension, hyperlipidemia, smoking, and prevalent cardiovascular disease (CVD). Participants with a prior stroke were excluded from the analyses where outcome was incident stroke; participants with prior CHD were excluded from the analyses where outcome was incident CHD. CVD refers to a combination of stroke and CHD. 


\section{을 \\ 길}

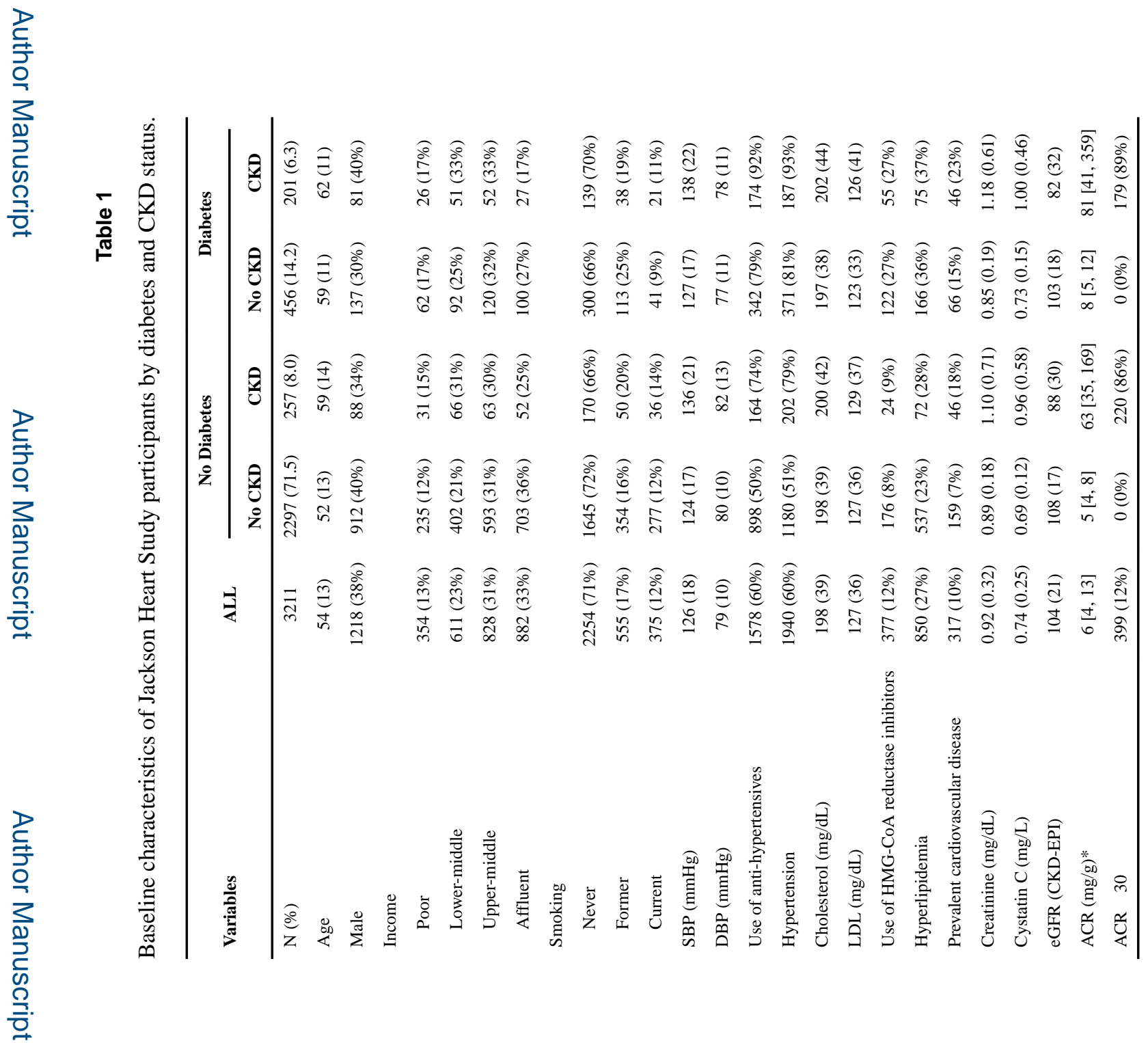




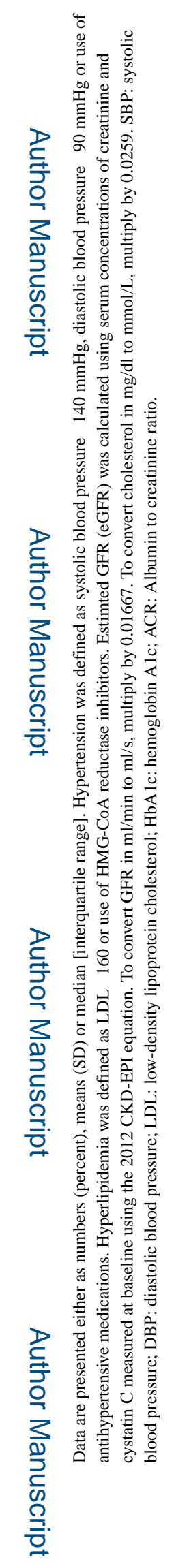




\section{Table 2}

Adjusted incidence rates of cardiovascular outcomes per 1000 person-years in people with and without diabetes in the Jackson Heart Study

\begin{tabular}{lccc}
\hline & $\mathbf{N}$ & Events & Adjusted incidence rates \\
\hline $\begin{array}{c}\text { Incident stroke } \\
\text { No Diabetes }\end{array}$ & 2473 & 35 & $1.3(0.6$ to 1.9$)$ \\
Diabetes & 615 & 36 & $6.5(3.8$ to 9.1$)$ \\
Incident CHD & & & \\
No Diabetes & 2401 & 48 & $1.9(1.1$ to 2.7$)$ \\
Diabetes & 571 & 33 & $6.9(4.1$ to 9.6$)$ \\
Cardiovascular Mortality & & \\
No Diabetes & 2554 & 52 & $1.3(0.6$ to 1.9$)$ \\
Diabetes & 657 & 46 & $6.0(3.7$ to 8.3$)$ \\
\hline
\end{tabular}

Incidence rates were calculated using Poisson regression and adjusted for age, age $^{2}$, gender, income, hypertension, hyperlipidemia, current smoking, and prevalent cardiovascular disease (CVD). Participants with a prior stroke (or CHD) were excluded from the analyses where outcome was incident stroke (or CHD). 

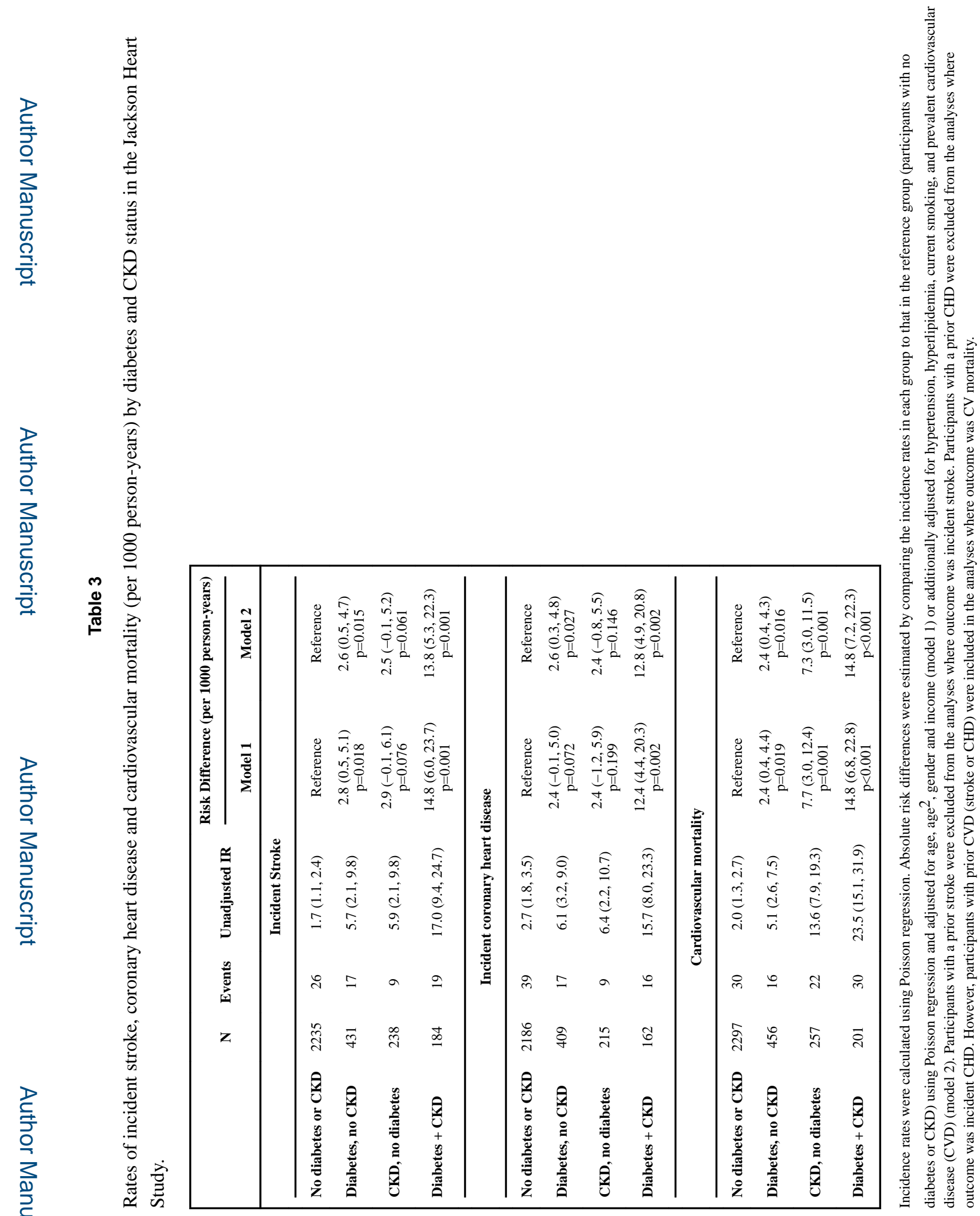

JAMA Cardiol. Author manuscript; available in PMC 2018 March 01. 


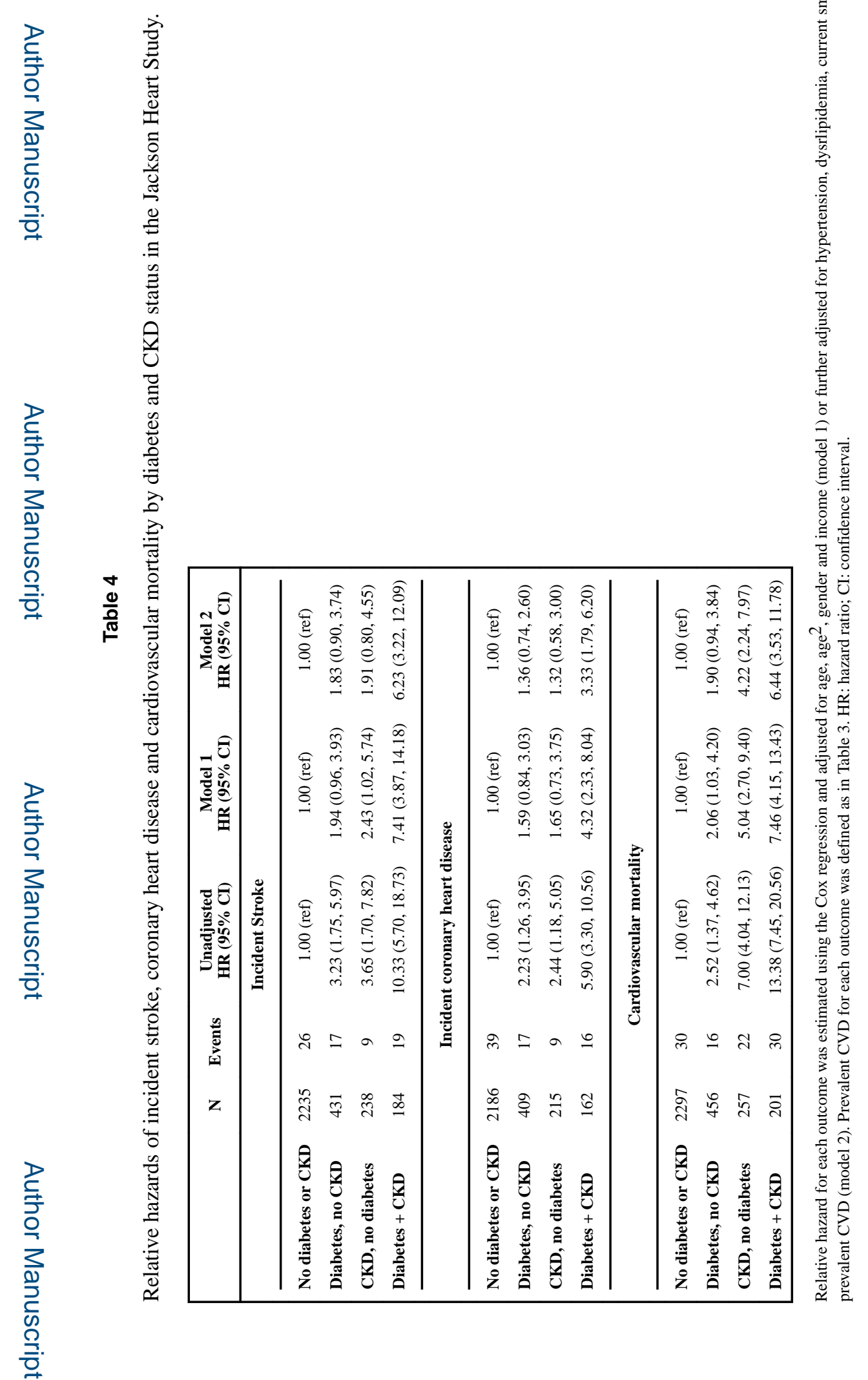

JAMA Cardiol. Author manuscript; available in PMC 2018 March 01. 